(1)

CrossMark

\title{
Impact of sleep disordered breathing on short-term post-operative outcome after elective coronary artery bypass graft surgery: a prospective observational study
}

\author{
Sven Rupprecht ${ }^{1,2}$, Torsten Schultze ${ }^{1}$, Andreas Nachtmann ${ }^{3}$, \\ Ardawan Julian Rastan ${ }^{4}$, Torsten Doenst ${ }^{5}$, Matthias Schwab ${ }^{1}$, Otto W. Witte ${ }^{1}$, \\ Sebastian Rohe ${ }^{1}$, Isabelle Zwacka ${ }^{1}$ and Heike Hoyer ${ }^{6}$
}

Affiliations: ${ }^{1}$ Hans-Berger Dept of Neurology, Jena University Hospital, Jena, Germany. ${ }^{2}$ Center for Sepsis Control and Care, Jena University Hospital, Jena, Germany. ${ }^{3}$ Dept of Neurology, Rotenburg Heart Centre, Rotenburg, Germany. ${ }^{4}$ Dept of Cardiothoracic Surgery, Rotenburg Heart Centre, Rotenburg, Germany. ${ }^{5}$ Dept of Cardiothoracic Surgery, Jena University Hospital, Jena, Germany. ${ }^{6}$ Institute of Medical Statistics, Information Sciences and Documentation, Jena University Hospital, Jena, Germany.

Correspondence: Sven Rupprecht, Hans-Berger Department of Neurology, Jena University Hospital, Erlanger Allee 101, D-07740 Jena, Germany. E-mail: sven.rupprechtamed.uni-jena.de

@ERSpublications

Sleep disordered breathing increases the rate of life-threatening post-operative complications after coronary surgery http://ow.ly/Ue25307U90Q

Cite this article as: Rupprecht S, Schultze T, Nachtmann A, et al. Impact of sleep disordered breathing on short-term post-operative outcome after elective coronary artery bypass graft surgery: a prospective observational study. Eur Respir J 2017; 49: 1601486 [https://doi.org/10.1183/13993003.01486-2016].

ABSTRACT Sleep disordered breathing (SDB) is common in patients with coronary disease, but its impact on post-operative recovery after coronary artery bypass graft surgery (CABG) is unclear. We therefore determined the effects of SDB on post-operative outcome after elective CABG.

In this prospective two-centre study, 219 patients due to receive elective CABG underwent cardiorespiratory polygraphy for $\mathrm{SDB}$ prior to surgery and were monitored for post-operative complications. The primary end-point was a composite of 30-day mortality or major post-operative complications (cardiac, respiratory, surgical, infectious, acute renal failure or stroke). Key secondary end-points were single components of the primary end-point.

SDB was present in $69 \%$ and moderate/severe SDB in $43 \%$ of the CABG patients. There was no difference in the composite of 30-day mortality or major postoperative complications between patients with and without SDB (OR 0.97, 95\% CI 0.49-1.96) and between patients with moderate/severe SDB and no/mild SDB (OR 1.07, 95\% CI 0.55-2.06). However, moderate/severe SDB was associated with higher rates of mortality (crude OR 10.1, 95\% CI 1.22-83.5), sepsis (OR 2.96, 95\% CI 1.17-7.50) and respiratory complications (OR 2.85, 95\% CI 1.46-5.55).

Although SDB was not associated with higher overall morbidity/mortality, moderate/severe SDB may increase the risk of death, and septic and respiratory complications, after elective CABG.

This article has supplementary material available from erj.ersjournals.com

Received: July 262016 | Accepted after revision: Dec 162016

This study is registered in the German Clinical Trial Register with identifier number DRKS00000704.

Support statement: The study was supported by the Federal Ministry of Education and Research (BMBF), Germany (FKZ: 01EO1002). Funding information for this article has been deposited with the Open Funder Registry.

Conflict of interest: Disclosures can be found alongside this article at erj.ersjournals.com

Copyright OERS 2017 


\section{Introduction}

Identifying high-risk patients remains a major challenge in coronary surgery. Sleep disordered breathing (SDB) is one of the most common chronic diseases (prevalence 50\%) in patients with coronary artery disease [1]. A growing body of evidence indicates that SDB increases the risk of major post-operative complications after abdominal and orthopaedic surgery [2]. A few studies have described an association between SDB and new-onset atrial fibrillation after coronary artery bypass graft surgery (CABG) [3], but the impact of SDB on major cardiac, respiratory, cerebral, surgical and infectious post-operative complications following coronary surgery is still unclear [4-6].

Here, we prospectively examined post-operative recovery in patients with stable coronary artery disease who underwent elective CABG. Our aims were 1) to evaluate whether SDB enhances postoperative morbidity, mortality and resource use; and 2) to identify specific SDB-related post-operative complications.

\section{Materials and methods}

The study was approved by the local ethical review committees (Jena University Hospital, number 2900-08/10; Hessian Medical Association, number MC 167/2011) and was registered in the German Clinical Trial Register (DRKS00000704). Written informed consent was obtained from all participants.

\section{Study design}

This was a prospective, controlled, observational two-centre study.

\section{Study subjects}

Consecutive patients with stable coronary artery disease who were scheduled for elective CABG at the Jena University Hospital, Germany, and at the Rotenburg Heart Centre, Germany, between 2011 and 2013 were recruited for the study. Exclusion criteria were SDB with continuous positive airway pressure (CPAP) therapy, and concomitant diseases with a major impact on post-operative outcome: unstable coronary artery disease or acute myocardial infarction, global respiratory failure, heart failure (New York Heart Association class III-IV), renal failure requiring dialysis, liver failure (Child-Pugh class C), immunosuppression (including AIDS and immunosuppressive therapy for any reason), alcohol dependency and cancer.

\section{Study protocol}

Patients were admitted to the sleep laboratory 2 days prior to surgery and underwent standardised overnight cardiorespiratory polygraphy, which included recording of oxygen saturation (finger pulse oximetry), oronasal airflow (nasal pressure transducer), respiratory effort (dual thoraco-abdominal respiratory inductance plethysmography belts), ECG and body position (fluid switch transducer) to determine the presence and severity of SDB.

Sleep apnoea was defined as obstructive apnoea/hypopnoea by either absence (apnoea) or a $\geqslant 30 \%$ reduction (hypopnoea) of tidal volume excursion and maintained thoraco-abdominal breathing effort for at least $10 \mathrm{~s}$ followed by $\geqslant 3 \%$ oxyhaemoglobin desaturation. Mixed apnoeas were classified as obstructive events due to their unknown origin. Central apnoea was defined as an absence of tidal volume and thoraco-abdominal breathing motion for at least $10 \mathrm{~s}$, and central hypopnoea by a $\geqslant 30 \%$ reduction in both tidal volume and thoraco-abdominal movement for at least $10 \mathrm{~s}$, with proportionate thoraco-abdominal in-phase movements followed by oxyhaemoglobin desaturation of $\geqslant 3 \%$ without snoring, increased inspiratory flattening of the nasal pressure flow signal, or out-of-phase thoraco-abdominal movements [7]. Total apnoea/hypopnoea index (AHI, number of events per hour in bed), obstructive AHI and central AHI were calculated and represent the number of events per hour. Given the low diagnostic performance of cardiorespiratory polygraphy in differentiating patients with no and mild SDB (indicated by a total AHI of $\geqslant 5 \mathrm{~h}^{-1}$ ) [8], the presence of SDB was defined by a total AHI of $\geqslant 10 \mathrm{~h}^{-1}$, and moderate/severe SDB by a total AHI of $\geqslant 20 \mathrm{~h}^{-1}$ [9]. Obstructive and central dominant SDB were defined by a total AHI of $\geqslant 10 \mathrm{~h}^{-1}$, and $\geqslant 50 \%$ of the relative number of obstructive or central events, respectively $[7,10]$.

Baseline characteristics documented for each patient included demographics, medication, comorbidities, neck circumference, and self-assessment of daytime sleepiness using the Epworth Sleepiness Scale (ESS) (a score of $\geqslant 11$ indicated daytime sleepiness). Surgical procedures and perioperative complications were obtained from standardised surgery and anaesthesia protocols recorded on the day of intervention. Patients were monitored continuously for potential post-operative complications for 0-7 days after surgery. A structured telephone interview with patients and/or relatives was carried out by the principal investigator (S. Rupprecht) on post-operative day 30 to assess survival, readmission to hospital, wound healing disturbances and reappearance of angina pectoris attacks. 


\section{End-points}

The primary composite endpoint comprised 30-day mortality and/or major post-operative complications, defined as death within 30 days and/or the occurrence of at least one of the following complications within 7 days of surgery: cardiac (re-animation, cardiogenic shock, myocardial ischaemia, implantation of a permanent pacemaker), respiratory (duration of mechanical ventilation $\geqslant 24 \mathrm{~h}$, re-intubation, tracheotomy), surgical (re-operation for any reason) and infectious (sepsis, deep wound infection, pneumonia), as well as acute renal failure requiring dialysis, or stroke.

Secondary end-points encompassed a detailed description of post-operative complications and resource use. These included cardiac, respiratory, neurological, surgical and infectious complications and represented a composite of several events (described in detail in supplementary table S2), in addition to hospital and 30-day mortality, acute renal failure, re-animation, systemic inflammatory response syndrome (SIRS), sepsis and septic shock. Resource use was determined by the length of post-operative stay in the intensive care unit (ICU) and hospital, number of transfers to the ICU, and duration and type of post-operative ventilatory support.

\section{Statistical analysis}

Sample size was calculated to achieve $80 \%$ power for the detection of an odds ratio of 6 [11] by comparing the rate of major post-operative complications in patients with and without SDB using a chi-squared test with a two-sided significance level of 5\%. Assuming an SDB prevalence of 50\% [1], and an event rate of $2.6 \%$ [12] in patients without SDB, 190 patients would have to be included in the study.

Baseline data were evaluated statistically by t-test, Mann-Whitney U-test or Fisher's exact test, depending on scale. Age, type of surgery and comorbidities were pre-defined as potential confounders of the association between SDB and end-points. The effects of SDB on the end-points was analysed using regression models depending on scale. In addition to SDB, covariates with a significant association with the end-point of interest at a significance level of 0.2 were selected for adjustment [13]. Finally, the following covariates were also considered for adjustment: age, body mass index (BMI), three-vessel coronary disease, ejection fraction, insulin-dependent diabetes, renal failure and on-pump surgery. Primary and binary secondary end-points were analysed using logistic regression models. Here, we determined the crude and adjusted odds ratios with 95\% confidence intervals and Wald test-based p-values. The number of independent variables in the logistic models, except for sepsis and septic shock, was restricted according to the rule of 10 events per variable [14] where essential confounders would otherwise be missing. In case of zero cell counts, we added 0.5 to each cell and reported only crude odds ratios. Frequencies of single components contributing to the composite end-points are presented without significance testing. Metric scale secondary end-points were analysed using generalised linear models assuming a gamma distribution and a log link function to account for the skewed distribution. The effects of SDB on metric end-points were estimated as crude and adjusted ratios of means with $95 \%$ confidence intervals and Wald test-based p-values. With the exception of confounder selection, the two-sided level of significance was 0.05. All data were managed in OpenClinica and analysed using the statistical software SAS 9.4.

\section{Results}

\section{Subjects}

From a total of 240 patients screened for eligibility, 221 were enrolled in the study. Of these, one died prior to surgery and one did not undergo surgery. The remaining 219 patients constituted the fully analysed dataset. One patient was subsequently lost to follow-up after discharge from hospital and was excluded from the 30-day mortality and primary composite end-point analyses. Patient flow is given in figure 1.

$\mathrm{SDB}$, defined as $\mathrm{AHI} \geqslant 10 \mathrm{~h}^{-1}$, was present in 151 patients, yielding a prevalence of $68.9 \%$ (95\% CI $62.4 \%-75.0 \%)$. Obstructive dominant SDB was present in 110 patients $(72.8 \%)$ and central dominant SDB in 41 patients $(27.2 \%)$. Baseline and polygraphic characteristics are summarised in tables 1 and 2. Patients with SDB were older $(\mathrm{p}=0.01)$ and had a higher BMI $(\mathrm{p}<0.01)$ and greater neck circumference $(\mathrm{p}<0.01)$ than those without SDB, but did not differ in the presence of subjective daytime sleepiness (ESS score $\geqslant 11 ; \mathrm{p}=0.35$ ). Patients with SDB had a slightly lower ejection fraction $(\mathrm{p}=0.04)$ and higher rate of three-vessel coronary heart disease $(p=0.02)$, insulin-dependent diabetes $(p=0.01)$ and chronic renal disease $(p=0.07)$ than those without.

\section{Coronary artery bypass graft surgery}

CABG procedures did not differ between patients with and without SDB (table 1). No differences were observed in operating room times or number of red cell transfusions between patients with and without SDB.

\section{End-points}

Primary end-point

A primary end-point event was observed in 21 (30.9\%) patients without and $48(32.0 \%)$ patients with $\mathrm{SDB}$, resulting in a confounder-adjusted odds ratio of 0.97 (95\% CI 0.48-1.96) (table 3). Frequencies of 


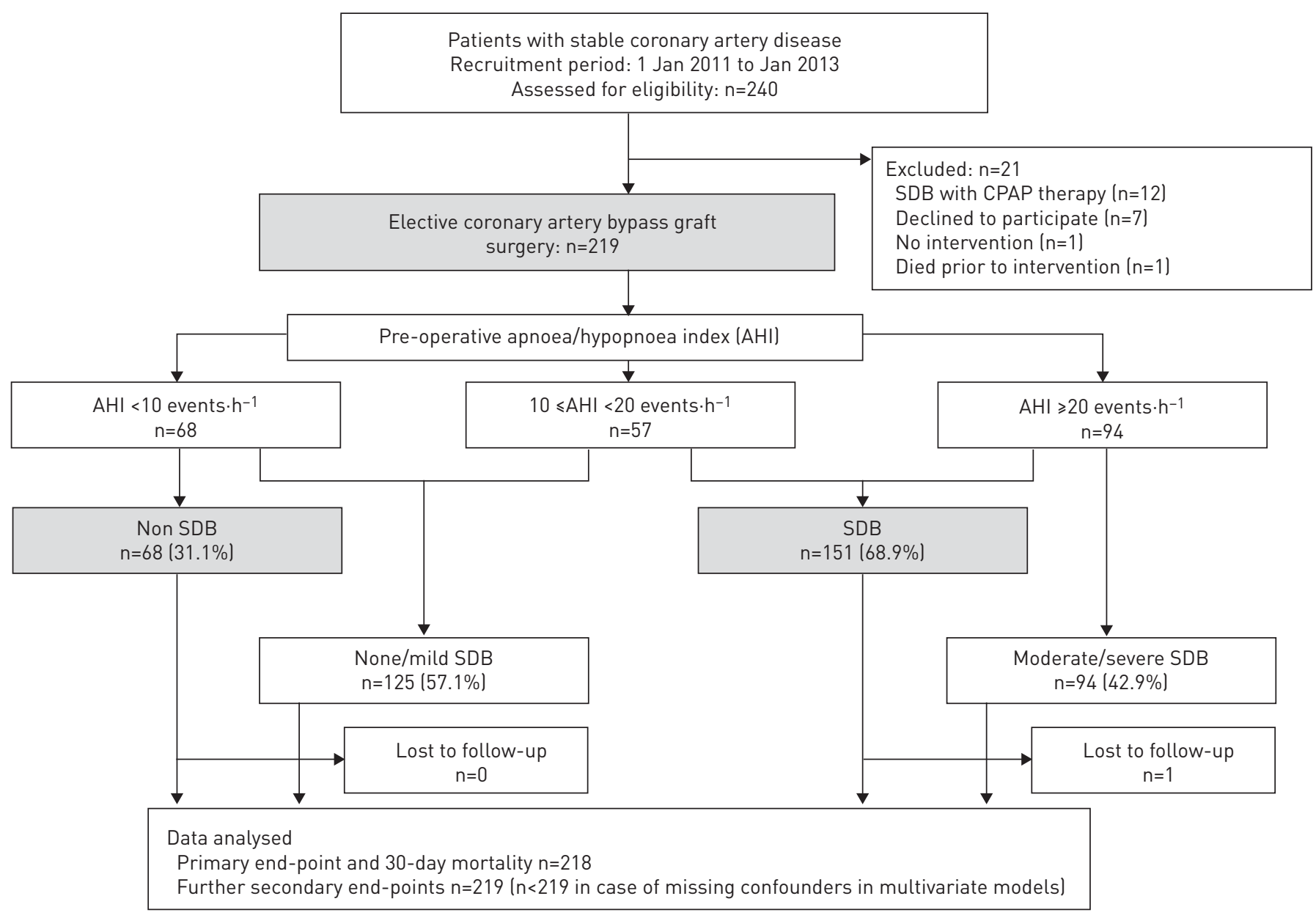

FIGURE 1 Patient flow.

single components contributing to the primary endpoint are shown in supplementary table S1. The most frequent single events were sepsis and cardiac ischaemia, which occurred in 26 (11.9\%) and 24 (11\%) patients, respectively.

\section{Secondary end-points}

Post-operative morbidity and mortality

All deceased patients had been diagnosed with SDB. Using zero-cell correction, the crude odds ratios were calculated as 7.1 (95\% CI 0.40-126) for hospital mortality and 8.2 (95\% CI 0.46-143) for 30-day mortality ( $p>0.05$ ). Adjusted ORs were not estimated because of the small number of events. Deceased patients were more likely to have had a lower ejection fraction $(\mathrm{p}<0.01)$ and chronic renal failure (creatinine level $>1.2 \mathrm{mg} \cdot \mathrm{dL}^{-1} ; \mathrm{p}=0.02$ ) than those who survived. These two potential confounders would probably reduce the strength of the SDB-mortality association if controlled for in the logistic regression model. Patients with SDB had more respiratory complications (adjusted OR 2.40, 95\% CI 1.15-4.97; table 3) with nonspecific desaturation events (33.8\% versus $19.1 \%$, supplementary table S2) as the major contributor. Additionally, the rate of cardiac complications tended to be elevated in the SDB group (adjusted OR 1.75, 95\% CI 0.93-3.27), driven by the higher rate of supraventricular arrhythmias (46.48\% versus $29.4 \%$, supplementary table S2). Although the rate of SIRS, sepsis and septic shock did not differ significantly, a trend towards a higher risk of sepsis (adjusted OR 2.24, 95\% CI 0.71-7.02) in patients with SDB was apparent. Rates of acute renal failure, re-animation and composite surgical, infectious and neurological complications did not differ significantly between patients with and without SDB (table 3).

\section{Resource use}

There was no statistically significant effect of SDB on post-operative length of stay in the ICU or hospital, duration of post-operative ventilation, or occurrence of ICU transfer after controlling for confounders (table 3). 
TABLE 1 Baseline and operative characteristics of patients undergoing elective coronary artery bypass graft surgery

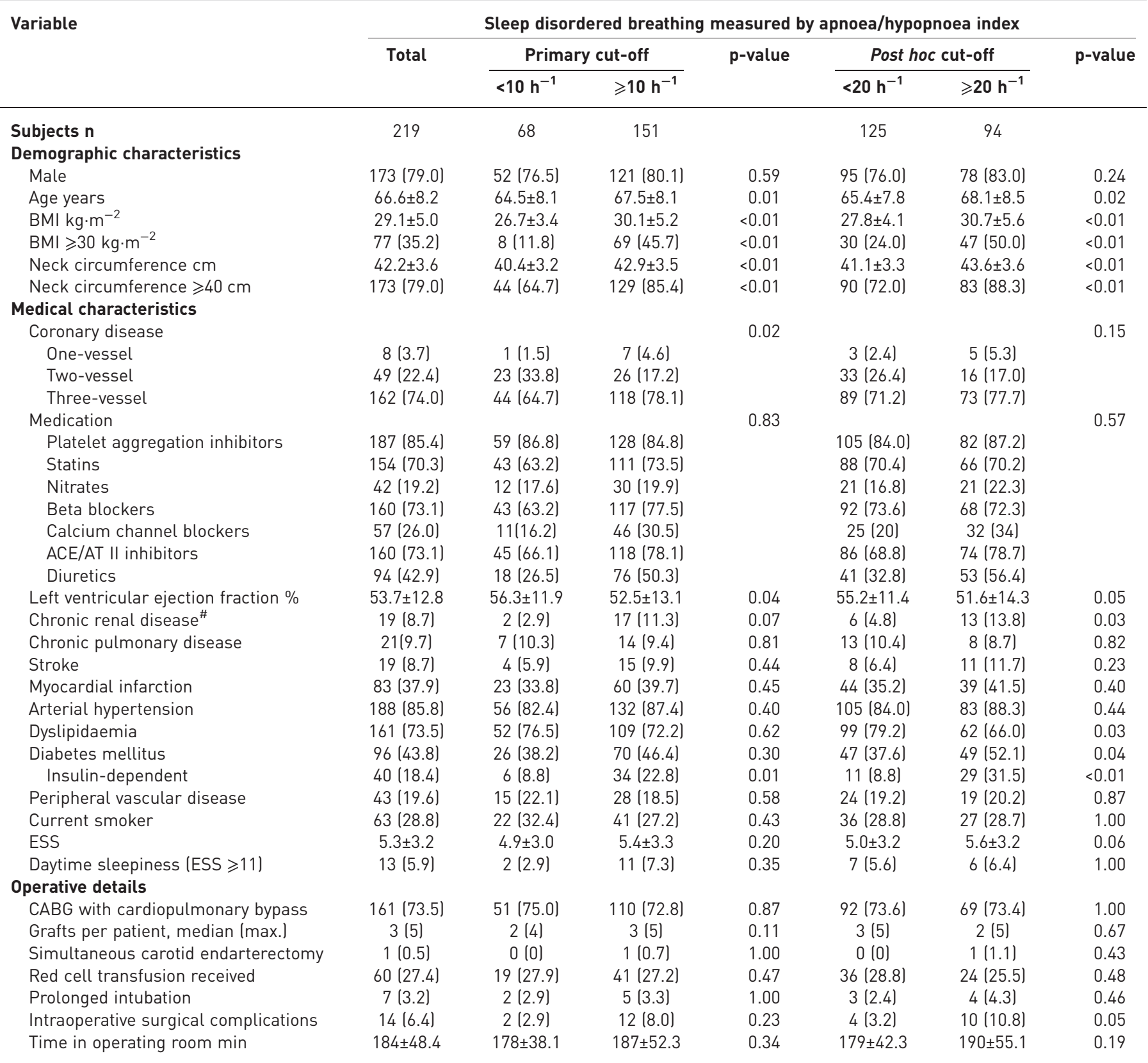

Data are reported as mean \pm SD or number (\%), unless otherwise indicated. BMI: body mass index; ACE: angiotensin-converting enzyme; AT II: angiotensin II; ESS: Epworth Sleepiness Scale; CABG: coronary artery bypass grafting. * ${ }^{\#}$ defined by a creatinine level $>1.2 \mathrm{mg} \cdot \mathrm{dL}^{-1}$.

Moderate/severe SDB (post hoc analysis)

To determine the impact of SDB severity on post-operative recovery, we analysed post-operative complication rates in patients with moderate/severe SDB $\left(\mathrm{AHI} \geqslant 20 \mathrm{~h}^{-1}\right)$ compared to no/mild SDB. Moderate/severe SDB was present in 94 of the 219 patients $(42.9 \%, 95 \%$ CI 36.3\%-49.8\%). Obstructive dominant SDB was present in 72 patients $(76.6 \%)$ and central dominant SDB in 22 patients (23.4\%) (table 2).

Demographic characteristics of patients with moderate/severe SDB were similar to those of all patients with SDB $\left(A H I \geqslant 10 \mathrm{~h}^{-1}\right)$. Patients with moderate/severe SDB showed a higher rate of comorbidities including diabetes mellitus $(p=0.04)$ and insulin-dependent diabetes mellitus $(p<0.01)$, chronic renal disease $(p=0.03)$ and dyslipidaemia $(p=0.03)$, and a slightly lower left ventricular ejection fraction $(\mathrm{p}=0.05)$ than patients with no/mild SDB (table 1$)$. CABG procedures did not differ between patients with moderate/severe SDB and no/mild SDB (table 1). 
TABLE 2 Polygraphic characteristics of patients undergoing elective coronary artery bypass graft surgery

\begin{tabular}{|c|c|c|c|c|c|}
\hline \multirow[t]{2}{*}{ Characteristics of polygraphy } & \multirow[t]{2}{*}{ Total } & \multicolumn{2}{|c|}{ Primary cut-off } & \multicolumn{2}{|c|}{ Post hoc cut-off } \\
\hline & & $<10 \mathrm{~h}^{-1}$ & $\geqslant 10 \mathrm{~h}^{-1}$ & $<20 \mathrm{~h}^{-1}$ & $\geqslant 20 \mathrm{~h}^{-1}$ \\
\hline Subjects $n$ & 219 & 68 & 151 & 125 & 94 \\
\hline Obstructive apnoea/hypopnoea index $\mathrm{h}^{-1}$ & $12.2 \pm 11.7$ & $2.9 \pm 1.8$ & $16.5 \pm 11.9$ & $5.5 \pm 4.2$ & $21.2 \pm 12.5$ \\
\hline Central apnoea/hypopnoea index $\mathrm{h}^{-1}$ & $6.9 \pm 6.8$ & $2.4 \pm 1.7$ & $8.9 \pm 7.3$ & $3.7 \pm 3.3$ & $11.1 \pm 8.0$ \\
\hline Obstructive dominant SDB ${ }^{\#}(\%)$ & & & $110(72.8)$ & & $72(76.6)$ \\
\hline
\end{tabular}

SDB: sleep disordered breathing. " : total apnoea/hypopnoea index $\geqslant$ cut-off value and obstructive apnoea/hypopnoea index > central apnoea/ hypopnoea index. ": total apnoea/hypopnoea index $\geqslant$ cut-off value and central apnoea/hypopnoea index > obstructive apnoea/hypopnoea index.

There was no difference in the primary composite end-point (adjusted OR 1.07, 95\% CI 0.55-2.06) between both groups (table 3). However, moderate/severe SDB was associated with higher hospital mortality (crude OR 8.45, 95\% CI 1.0-71.5) and 30-day mortality (crude OR 10.1, 95\% CI 1.22-83.5).

TABLE 3 Effect of sleep disordered breathing on post-operative outcome

Binary end points

Sleep disordered breathing measured by apnoea/hypopnoea index

\begin{tabular}{|c|c|c|c|c|c|c|c|c|}
\hline & \multicolumn{4}{|c|}{ Primary cut-off: $<10 \mathrm{~h}^{-1}$ versus $\geqslant 10 \mathrm{~h}^{-1}$} & \multicolumn{4}{|c|}{ Post hoc cut-off: $<20 \mathrm{~h}^{-1}$ versus $\geqslant 20 \mathrm{~h}^{-1}$} \\
\hline & $\begin{array}{c}\text { Crude } \\
\text { OR }(95 \% \text { CI })\end{array}$ & p-value & $\begin{array}{c}\text { Adjusted } \\
\operatorname{OR}^{\mathrm{d}}(95 \% \mathrm{Cl})\end{array}$ & p-value & $\begin{array}{c}\text { Crude } \\
\text { OR }(95 \% \mathrm{CI})\end{array}$ & p-value & $\begin{array}{c}\text { Adjusted } \\
\text { OR }^{\text {d }}(95 \% \mathrm{CI})\end{array}$ & p-value \\
\hline 30-day mortality ${ }^{c}$ & $8.17(0.46-143.4)^{b}$ & 0.15 & & & $10.1(1.22-83.5)$ & 0.03 & & \\
\hline Hospital mortality ${ }^{c}$ & $7.11(0.40-126.2)^{b}$ & 0.18 & & & $8.45(1.00-71.5)$ & 0.05 & & \\
\hline Neurological complications ${ }^{a}$ & $1.34(0.62-2.86)$ & 0.45 & $0.97(0.42-2.22)^{4}$ & 0.95 & $2.04(1.03-4.03)$ & 0.04 & $1.55(0.73-3.27)^{4}$ & 0.25 \\
\hline Surgical complications ${ }^{a}$ & $0.55(0.19-1.56)$ & 0.26 & $0.78(0.26-2.29)^{5}$ & 0.65 & $0.42(0.13-1.35)$ & 0.14 & $0.57(0.17-1.88)^{5}$ & 0.35 \\
\hline Acute renal failure ${ }^{c}$ & $3.23(0.16-63.4)^{\mathrm{b}}$ & 0.44 & & & $9.60^{\mathrm{b}}(0.49-188)$ & 0.14 & & \\
\hline Infectious complications ${ }^{a}$ & $2.02(0.87-4.65)$ & 0.10 & $1.69(0.71-4.00)^{6}$ & 0.23 & $2.06(1.02-4.13)$ & 0.04 & $1.77(0.84-3.70)^{6}$ & 0.13 \\
\hline $\mathrm{SIRS}^{\mathrm{c}}$ & $1.25(0.40-3.89)$ & 0.70 & & & $1.00(0.33-3.00)$ & 1.00 & & \\
\hline Sepsis & $2.73(0.90-8.25)$ & 0.08 & $2.24(0.71-7.02)^{7}$ & 0.17 & $3.46(1.43-8.37)$ & $<0.01$ & $2.96(1.17-7.50)^{7}$ & 0.02 \\
\hline Metric end points & $\begin{array}{l}\text { Crude mean } \\
\text { ratio }(95 \% \mathrm{CI})\end{array}$ & p-value & $\begin{array}{l}\text { Adjusted mean } \\
\text { ratio }\left(95 \% \mathrm{CI}^{\mathrm{d}}\right.\end{array}$ & p-value & $\begin{array}{l}\text { Crude mean } \\
\text { ratio }(95 \% \mathrm{CI})\end{array}$ & p-value & $\begin{array}{l}\text { Adjusted mean } \\
\text { ratio }(95 \% \mathrm{CI})^{\mathrm{d}}\end{array}$ & p-value \\
\hline Post-operative hou & $1.35(1.06-1.72)$ & 0.01 & $1.15(0.90-1.47)^{10}$ & 0.25 & $1.34(1.07-1.68)$ & 0.01 & $1.18(0.94-1.48)^{10}$ & 0.15 \\
\hline Post-operative days in hospital & $1.10(0.97-1.24)$ & 0.12 & $1.00(0.89-1.12)^{11}$ & 0.98 & $1.07(0.96-1.20)$ & 0.19 & $0.96(0.85-1.08)^{11}$ & 0.48 \\
\hline Hours of invasive ventilation & $1.30(0.99-1.70)$ & 0.05 & $0.93(0.71-1.22)^{12}$ & 0.60 & $1.62(1.27-2.08)$ & $<0.01$ & $1.13(0.86-1.48)^{12}$ & 0.38 \\
\hline Hours of oxygen supply & $1.19(0.99-1.43)$ & 0.06 & $1.15(0.96-1.36)^{13 a}$ & 0.12 & $1.16(0.97-1.37)$ & 0.10 & $1.06(0.89-1.25)^{13 b}$ & 0.50 \\
\hline Hours of noninvasive ventilation & $1.82(1.03-3.19)$ & 0.04 & $1.59(0.87-2.89)^{14 a}$ & 0.13 & $1.48(0.87-2.50)$ & 0.14 & $0.61(0.33-1.12)^{14 b}$ & 0.11 \\
\hline
\end{tabular}

In principle, seven covariates were considered for adjustment: age, three-vessel coronary heart disease (CHD), insulin-dependent diabetes, creatinine level $>1.2 \mathrm{mg} \cdot \mathrm{dL}^{-1}$, on-pump surgery, body mass index, ejection fraction. SIRS: systemic inflammatory response syndrome; ICU: intensive care unit. ${ }^{a}$ : composite endpoint; ${ }^{b}$ : owing to zero cell count, odds ratio was calculated by adding 0.5 to each cell; ${ }^{c}$ : confounder adjustment was omitted owing to too few or too many events; ${ }^{d}$ : adjusted for the following: ${ }^{1}$ :age, body mass index, creatinine level $>1.2 \mathrm{mg} \cdot \mathrm{dL}^{-1}$, on-pump surgery, ejection fraction; ${ }^{2}$ : age, three-vessel $\mathrm{CHD} ;{ }^{3}$ : age, insulin-dependent diabetes, creatinine level $>1.2 \mathrm{mg} \cdot \mathrm{dL}^{-1}$, on-pump surgery; ${ }^{4}$ : age, creatinine level $>1.2 \mathrm{mg} \cdot \mathrm{dL}^{-1}$, on-pump surgery; ${ }^{5}$ : body mass index; ${ }^{6}$ : three-vessel $\mathrm{CHD}$, insulin-dependent diabetes; ${ }^{7}$ : on-pump surgery, ejection fraction, creatinine level $>1.2 \mathrm{mg} \cdot \mathrm{dL}^{-1} ;{ }^{8}$ : on-pump surgery, ejection fraction, creatinine level $>1.2 \mathrm{mg} \cdot \mathrm{dL}^{-1}$; ${ }^{9}$ : on-pump surgery, three-vessel CHD, creatinine level $>1.2 \mathrm{mg} \cdot \mathrm{dL}^{-1} ;{ }^{10}$ : age, three-vessel CHD; ${ }^{11}$ : age, three-vessel CHD, insulin-dependent diabetes, creatinine level $>1.2 \mathrm{mg} \cdot \mathrm{dL}^{-1} ;{ }^{12}$ : age, three-vessel $\mathrm{CHD}$, creatinine level $>1.2 \mathrm{mg} \cdot \mathrm{dL}^{-1}$; on-pump surgery, body mass index; ${ }^{13 a}$ : age, three-vessel CHD, ejection fraction; ${ }^{13 b}$ : age, three-vessel CHD, ejection fraction, insulin-dependent diabetes; ${ }^{14 a}$ : age, on-pump surgery, body mass index; ${ }^{14 \mathrm{~b}}$ : age, on-pump surgery. 
Significantly higher rates of sepsis (adjusted OR 2.96, 95\% CI 1.17-7.50) and respiratory complications (adjusted OR 2.85, 95\% CI 1.46-5.55) were present in patients with moderate/severe SDB (table 2). When the different origins of SDB were considered, the adjusted OR for sepsis was 5.74 (95\% CI 1.54-21.45) for central dominant and 2.80 (95\% CI 1.07-7.34) for obstructive dominant moderate/severe SDB compared to no/mild SDB (adjusted for on-pump surgery and ejection fraction). For respiratory complications we found an adjusted OR of 5.51 (95\% CI 1.88-16.13) in the presence of central dominant SDB and an OR of 2.28 (95\% CI 1.11-4.70) in the presence of obstructive dominant SDB (adjusted for age, insulin-dependent diabetes and creatinine level $\left.>1.2 \mathrm{mg} \cdot \mathrm{dL}^{-1}\right)$. Respiratory complications in patients with moderate/severe SDB were mainly due to the higher rate of acute hypoxaemia attributable to pneumonia (11.7\% versus 2.4\%) and nonspecific desaturation events (38.3\% versus 22.4\%). Septic shock (adjusted OR 2.53, 95\% CI 0.86-7.37) and the composites of neurological (adjusted OR 1.55, 95\% CI 0.73-3.27) and infectious (adjusted OR 1.77, 95\% CI 0.84-3.70) complications were more frequent in patients with moderate/severe SDB than in those without, but were not significantly increased after adjusting for confounders. Frequent neurological complications were sopor and coma (25.5\% versus $13.6 \%)$ and the most frequent infectious complication was pneumonia (11.7\% versus $3.2 \%)$. All three patients with acute renal failure suffered from moderate/severe SDB (zero-cell-corrected crude OR 9.60, 95\% CI 0.49-188) (table 2). Resource use did not differ significantly after adjusting for confounders.

\section{Discussion}

Although SDB is highly prevalent in patients with stable coronary artery disease undergoing elective CABG, it had no effect on the overall rate of major post-operative morbidity/mortality in the present study. However, SDB was associated with increased mortality and a higher rate of sepsis and pneumonia. These post-operative complications were more apparent in patients with moderate/severe SDB (AHI $\geqslant 20 \mathrm{~h}^{-1}$ ) compared to those with any $\operatorname{SDB}\left(\mathrm{AHI} \geqslant 10 \mathrm{~h}^{-1}\right)$. In addition, septic and respiratory complications were more frequent in patients with central dominant SDB than in those with obstructive dominant SDB.

The global effect of SDB on post-operative morbidity/mortality in our study was assessed either by the overall rate of death within 30 days of surgery, or by major cardiac, respiratory, renal, surgical or neurological adverse events as the primary composite end-point [15]. Interestingly, we found no association between SDB and the overall rate of morbidity/mortality after elective CABG. Our global description of post-operative morbidity/mortality might not adequately reflect SDB-specific effects on post-operative morbidity [16]. Correspondingly, we observed higher rates of sepsis, septic shock and infectious respiratory complications in patients with SDB, which seem to represent more specific end-points for assessing post-operative outcome after CABG in the presence of SDB.

Regarding the different origins of SDB, we observed a higher incidence of septic and respiratory complications in patients with central dominant SDB than those with obstructive dominant SDB. The associations between central dominant SDB and septic and respiratory complications were independent of cardiac output (left ventricular ejection fraction) in the multivariate analysis; this indicates that the increased risk is mediated predominantly by the central dominant SDB itself and not by the impairment in left ventricular function. Our findings are in agreement with an earlier population-based study that showed that central dominant, not obstructive dominant, SDB predicts mortality in the normal elderly population [17]. Since both central and obstructive dominant SDB are also independent risk factors for poor prognosis in heart failure, a clear distinction of SDB subtypes is essential in subsequent SDB-related post-operative outcome studies [18, 19].

The various SDB-specific effects on the immune, endothelial and autonomic nervous systems may explain the higher incidence of sepsis in our SDB patients. For instance, repetitive apnoea results in intermittent hypoxia and sympathetic overactivity with manifold systemic consequences including a pro-inflammatory state, hypercoagulability, oxidative stress, endothelial dysfunction and impaired vasoreactivity [20]. All these conditions play a major role in the pathogenesis of sepsis [21]. It is possible that an SDB-related pro-inflammatory state, sympathetic overactivity and hypercoagulability boost the post-operative inflammatory response, whereas oxidative stress and impaired vasoreactivity facilitate microvascular disturbances and thus enhance susceptibility to, and severity of, sepsis [20,21].

Pneumonia was the most frequent respiratory and infectious complication in our SDB patients. The elevated incidence of post-operative pneumonia is probably due to a higher rate of pulmonary aspiration, post-operative atelectasis and pulmonary oedema, factors that are frequently observed in SDB patients [2]. The heightened pro-inflammatory state in SDB may further boost the pulmonary inflammatory process [22].

In our study, patients with SDB showed higher hospital and 30-day mortality. Notably, seven of the nine patients died due to sepsis or septic shock following surgery. Since SDB was associated with a higher rate of sepsis, adequate treatment of SDB may well decrease the post-operative rate of sepsis, and thus mortality. 
CPAP ventilation is the most effective treatment option in SDB [23]. In acute hypoxic patients, early use of CPAP in the immediate post-operative setting reduces the rate of pneumonia and sepsis after major abdominal surgery [24]. Since acute post-operative hypoxaemia is mainly due to undiagnosed SDB [2, 25], it is safe to assume that post-operative CPAP treatment could also be beneficial for SDB patients. Indeed, it has been shown that CPAP reduces inflammatory state [26], oxidative stress [27], sympathetic overactivity [28] and hypercoagulability [29], and improves microvascular function [27]. Whilst administration of CPAP even for one night reduces sympathetic activity [28], its other beneficial effects mentioned above are detectable only at about 2 weeks after treatment initiation $[27,29,30]$. To maximise CPAP therapy benefits, future studies should clarify optimal treatment initiation: for example, during the pre-operative or immediate post-operative period. Furthermore, given the higher incidence of post-operative sepsis and pneumonia in patients with SDB, pre-operative SDB screening would allow improved risk stratification and preventive CPAP intervention.

We used cardiorespiratory polygraphy to estimate the prevalence and severity of SDB in our study cohort; this is the recommended screening tool in high-risk populations of moderate/severe SDB [31]. However, cardiorespiratory polygraphy is poorly accessible and requires qualified medical personnel, factors that could limit its use as a routine clinical screening tool. Taking this important issue into account, we also evaluated more easily applicable standard SDB screening methods, such as determination of BMI and neck circumference, and self-assessment of sleepiness using the ESS questionnaire. Applying the recommended cut-offs for screening (ESS score $\geqslant 11$, neck circumference $>40 \mathrm{~cm}$, BMI $>30$ ), moderate/severe SDB would be detected with a sensitivity of $6.4 \%, 88.3 \%$ and $50 \%$, respectively (table 1). The corresponding specificities are $94.6 \%, 28 \%$ and $76 \%$. Since a comparable low diagnostic performance has also been reported for other SDB questionnaires [32] and anthropometric measurements [32, 33], cardiorespiratory polygraphy should be considered as the primary screening tool in pre-operative SDB risk assessment.

The strengths of our study include the prospective and consecutive enrolment of patients in two study centres, the use of a pre-calculated sample size for analysing the primary end-point, and the adoption of an adequate observational period to address our study questions. Additional strengths were the inclusion of the gold standard for SDB screening (involving standardised overnight cardiorespiratory polygraphy prior to surgery) and ensuring the highest scientific standards by adhering strictly to the study protocol regarding patient enrolment, data collection and analysis. Finally, CABG is a highly standardised surgical procedure that reduces potential bias of different surgeons and surgical procedures on post-operative outcome.

However, the study also had some limitations. In our study, the primary analysis was based on an AHI cut-off point of $\geqslant 10 \mathrm{~h}^{-1}$, which separates patients with mild to severe SDB from those without relevant SDB. Except for respiratory complications, our primary analyses failed to show statistically significant associations between SDB and major post-operative complications. The increase in post-operative risk of sepsis was statistically significant only in patients with moderate/severe SDB (i.e. once the AHI cut-off was changed from $10 \mathrm{~h}^{-1}$ to $20 \mathrm{~h}^{-1}$ ). Even if the new cut-off could be justified by the unexpectedly high SDB prevalence of $69 \%$ for an $\mathrm{AHI}$ of $\geqslant 10 \mathrm{~h}^{-1}$, results of the post hoc analysis should be interpreted with care and need to be validated. However, in agreement with our data, several longitudinal studies found an increase in vascular risk and overall mortality in moderate/severe, but not mild, SDB [34, 35]. We initially pre-defined age, type of surgery and comorbidities as potential confounders in our study protocol. Subsequently, we identified the relevant comorbidities after exploring baseline data and specified a list of seven candidates to control for confounding. According to the rule of 10 events per variable in logistic regression models, we restricted the maximum number of covariates depending on the observed number of binary end-point events. However, for rare or very frequent endpoints, such as mortality, acute renal failure, SIRS, re-animation and ICU transfer, only crude odds ratios were estimated. Therefore, residual confounding could be present for these end-points.

In conclusion, our findings indicate that moderate/severe SDB is an independent risk factor for severe post-operative complications in patients undergoing elective CABG. Until more data are available, moderate/ severe SDB should be considered as an increased post-operative risk factor for sepsis and respiratory complications. Since SDB is treatable, further studies are needed to determine whether pre-operative polygraph screening for SDB, and subsequent therapy, improve patient outcome after CABG.

\section{Acknowledgements}

We thank all patients who participated in the study. We also thank Cornelia Eichhorn for data management support and Nasim Kroegel for language editing.

Author contributions: S. Rupprecht was the principal investigator and takes full responsibility for the integrity of the data and the accuracy of the analysis; S. Rupprecht and H. Hoyer conceived the study and drafted the manuscript; H. Hoyer performed the statistical analysis; T. Schultze performed and analysed the polygraphic recordings; S. Rohe and I. Zwacka recruited the patients and performed the polygraphic recordings; A. Nachtmann, T. Doenst, A. Rastan, 
O.W. Witte and M. Schwab interpreted the data and revised the manuscript. The corresponding author confirms that this manuscript is an honest, accurate and transparent account of the study being reported; that no important aspects of the study have been omitted; and that any discrepancies from the study as planned have been explained.

\section{References}

1 Prinz C, Bitter T, Piper C, et al. Sleep apnea is common in patients with coronary artery disease. Wien Med Wochenschr 2010; 160: 349-355.

2 Opperer M, Cozowicz C, Bugada D, et al. Does obstructive sleep apnea influence perioperative outcome? A qualitative systematic review for the Society of Anesthesia and Sleep Medicine Task Force on preoperative preparation of patients with sleep-disordered breathing. Anesth Analg 2016; 122: 1321-1334.

3 Qaddoura A, Kabali C, Drew D, et al. Obstructive sleep apnea as a predictor of atrial fibrillation after coronary artery bypass grafting: a systematic review and meta-analysis. Can J Cardiol 2014; 30: 1516-1522.

4 Uchôa CH, Danzi-Soares NJ, Nunes FS, et al. Impact of OSA on cardiovascular events after coronary artery bypass surgery. Chest 2015; 147: 1352-1360.

5 Amra B, Niknam N, Sadeghi MM, et al. Obstructive sleep apnea and postoperative complications in patients undergoing coronary artery bypass graft surgery: a need for preventive strategies. Int J Prev Med 2014; 5: $1446-1451$

6 Kua J, Zhao LP, Kofidis T, et al. Sleep apnoea is a risk factor for acute kidney injury after coronary artery bypass grafting. Eur J Cardiothorac Surg 2016; 49: 1188-1194.

7 Berry RB, Budhiraja R, Gottlieb DJ, et al. Rules for scoring respiratory events in sleep: update of the 2007 AASM Manual for the Scoring of Sleep and Associated Events. Deliberations of the Sleep Apnea Definitions Task Force of the American Academy of Sleep Medicine. J Clin Sleep Med 2012; 8: 597-619.

8 El Shayeb M, Topfer LA, Stafinski T, et al. Diagnostic accuracy of level 3 portable sleep tests versus level 1 polysomnography for sleep-disordered breathing: a systematic review and meta-analysis. CMAJ 2014; 186: E25-E51.

9 Gross JB, Bachenberg KL, Benumof JL, et al. Practice guidelines for the perioperative management of patients with obstructive sleep apnea: a report by the American Society of Anesthesiologists Task Force on perioperative management of patients with obstructive sleep apnea. Anesthesiology 2006; 104: 1081-1093.

10 Iber C, Ancoli-Israel S, Chesson A, et al. for the American Academy of Sleep Medicine. The AASM Manual for the Scoring of Sleep and Associated Events: Rules, Terminology and Technical Specifications. 1st Edn. Westchester, Illinois, American Academy of Sleep Medicine, 2007.

11 Kaw R, Pasupuleti V, Walker E, et al. Postoperative complications in patients with obstructive sleep apnea. Chest 2012; 141: 436-441.

12 Motomura N, Miyata $\mathrm{H}$, Tsukihara $\mathrm{H}$, et al. First report on 30-day and operative mortality in risk model of isolated coronary artery bypass grafting in Japan. Ann Thorac Surg 2008; 86: 1866-1872.

13 Maldonado G, Greenland S. Simulation study of confounder-selection strategies. Am J Epidemiol 1993; 138: 923-936.

14 Peduzzi P, Concato J, Feinstein AR, et al. Importance of events per independent variable in proportional hazards regression analysis. II. Accuracy and precision of regression estimates. J Clin Epidemiol 1995; 48: 1503-1510.

15 Shroyer AL, Grover FL, Hattler B, et al. On-pump versus off-pump coronary-artery bypass surgery. N Engl J Med 2009; 361: 1827-1837.

16 Montori VM, Permanyer-Miralda G, Ferreira-Gonzalez I, et al. Validity of composite end points in clinical trials. BMJ 2005; 330: 594-596.

17 Johansson $\mathrm{P}$, Alehagen U, Svanborg E, et al. Clinical characteristics and mortality risk in relation to obstructive and central sleep apnoea in community-dwelling elderly individuals: a 7-year follow-up. Age Ageing 2012; 41: 468-474.

18 Bitter T, Westerheide N, Prinz C, et al. Cheyne-Stokes respiration and obstructive sleep apnoea are independent risk factors for malignant ventricular arrhythmias requiring appropriate cardioverter-defibrillator therapies in patients with congestive heart failure. Eur Heart J 2011; 32: 61-74.

19 Javaheri S, Shukla R, Zeigler H, et al. Central sleep apnea, right ventricular dysfunction, and low diastolic blood pressure are predictors of mortality in systolic heart failure. J Am Coll Cardiol 2007; 49: 2028-2034.

20 Calvin AD, Somers VK. Obstructive sleep apnea and cardiovascular disease. Curr Opin Cardiol 2009; 24: 516-520.

21 Rittirsch D, Flierl MA, Ward PA. Harmful molecular mechanisms in sepsis. Nat Rev Immunol 2008; 8: 776-787.

22 Ryan S, Taylor CT, McNicholas WT. Selective activation of inflammatory pathways by intermittent hypoxia in obstructive sleep apnea syndrome. Circulation 2005; 112: 2660-2667.

23 Giles TL, Lasserson TJ, Smith BH, et al. Continuous positive airways pressure for obstructive sleep apnoea in adults. Cochrane Database Syst Rev 2006; CD001106.

24 Squadrone V, Coha M, Cerutti E, et al. Continuous positive airway pressure for treatment of postoperative hypoxemia: a randomized controlled trial. JAMA 2005; 293: 589-595.

25 Finkel KJ, Searleman AC, Tymkew H, et al. Prevalence of undiagnosed obstructive sleep apnea among adult surgical patients in an academic medical center. Sleep Med 2009; 10: 753-758.

26 Baessler A, Nadeem R, Harvey M, et al. Treatment for sleep apnea by continuous positive airway pressure improves levels of inflammatory markers - a meta-analysis. J Inflamm (Lond) 2013; 10: 13.

27 Büchner NJ, Quack I, Woznowski M, et al. Microvascular endothelial dysfunction in obstructive sleep apnea is caused by oxidative stress and improved by continuous positive airway pressure therapy. Respiration 2011; 82: 409-417.

28 Noda A, Nakata S, Koike Y, et al. Continuous positive airway pressure improves daytime baroreflex sensitivity and nitric oxide production in patients with moderate to severe obstructive sleep apnea syndrome. Hypertens Res 2007; 30: 669-676.

29 Phillips CL, McEwen BJ, Morel-Kopp MC, et al. Effects of continuous positive airway pressure on coagulability in obstructive sleep apnoea: a randomised, placebo-controlled crossover study. Thorax 2012; 67: 639-644.

30 Ip MS, Tse HF, Lam B, et al. Endothelial function in obstructive sleep apnea and response to treatment. Am J Respir Crit Care Med 2004; 169: 348-353.

31 Collop NA, Anderson WM, Boehlecke B, et al. Clinical guidelines for the use of unattended portable monitors in the diagnosis of obstructive sleep apnea in adult patients. Portable Monitoring Task Force of the American Academy of Sleep Medicine. J Clin Sleep Med 2007; 3: 737-747. 
32 Abrishami A, Khajehdehi A, Chung F. A systematic review of screening questionnaires for obstructive sleep apnea. Can J Anaesth 2010; 57: 423-438.

33 Boynton G, Vahabzadeh A, Hammoud S, et al. Validation of the STOP-BANG questionnaire among patients referred for suspected obstructive sleep apnea. J Sleep Disord Treat Care 2013; 2.

34 Marshall NS, Wong KK, Cullen SR, et al. Sleep apnea and 20-year follow-up for all-cause mortality, stroke, and cancer incidence and mortality in the Busselton health study cohort. J Clin Sleep Med 2014; 10: 355-362.

35 Martínez-García MA, Campos-Rodríguez F, Catalán-Serra P, et al. Cardiovascular mortality in obstructive sleep apnea in the elderly: role of long-term continuous positive airway pressure treatment: a prospective observational study. Am J Respir Crit Care Med 2012; 186: 909-916. 